Acknowledgements: We would like to thank all patients and rheumatologists contributing to the SCQM registry, as well as the entire SCQM staff. A list of rheumatology offices and hospitals which contribute to the SCQM registry can be found at http://www.scqm.ch/institutions. The SCQM is financially supported by pharmaceutical industries and donors. A list of financial supporters can be found at http://www.scqm.ch/sponsors.

Disclosure of Interests: Enriqueta Vallejo-Yagüe: None declared, Theresa Burkard: None declared, Burkhard Moeller Speakers bureau: AbbVie, Bristol Myers, Eli Lilly, Janssen, Pfizer, Roche, Novartis, Merck, Axel Finckh Speakers bureau: Pfizer, Eli-Lilly, Paid instructor for: Pfizer, Eli-Lilly, Consultant of: AbbVie, AB2Bio, BMS, Gilead, Pfizer, Viatris, Grant/research support from: Pfizer, BMS, Novartis, Andrea Michelle Burden: None declared

DOI: 10.1136/annrheumdis-2021-eular.1348

\section{POS0213 20 YEAR FOLLOW-UP OF CARDIOVASCULAR EVENT RISK IN RHEUMATOID ARTHRITIS COMPARED TO DIABETES}

R. Raadsen ${ }^{1}$, R. Agca ${ }^{1,2}$, A. Voskuyl $\left.\right|^{2}$, M. Boers ${ }^{3}$, W. Lems $^{1,2}$,

M. Nurmohamed ${ }^{1,2} .{ }^{1}$ Amsterdam Rheumatology and Immunology Center, location Reade, Rheumatology, Amsterdam, Netherlands; ${ }^{2}$ Amsterdam Rheumatology and Immunology Center, location VU University Medical Center, Rheumatology, Amsterdam, Netherlands; ${ }^{3}$ Amsterdam University Medical Center, Location VU University Medical Center, Biostatistics and Epidemiology, Amsterdam, Netherlands

Background: Patients with rheumatoid arthritis have an increased risk for developing cardiovascular diseases (CVD) compared to the general population, similar to the CVD risk in patients with diabetes mellitus. However, there are no controlled studies investigating the incidence of cardiovascular (CV) events in RA patients with follow up of more than 20 years.

Objectives: The objectives of the current study were to investigate the incidence rates of CV events in a long-term follow up cohort of RA patients, and to compare these to a similar cohort representing the general population, ie. The Hoorn study.

Methods: The CARRÉ study is an ongoing prospective cohort study, which started in 2001, investigating CV mortality and morbidity in 353 randomly selected patients with RA. Primary endpoints, i.e. verified medical history of coronary, cerebral or peripheral arterial disease, were determined at baseline, and after three, ten, fifteen and twenty years of follow up. Patients were censored at the date of an experienced CV event or their death. Incidence density rates per 100 patient years were calculated. Data were compared to results from the Hoorn study, a Dutch cohort study of glucose metabolism and other CV risk factors that began in 1989. All 2,484 participants were subject to an extensive and repeated CV screening program similar to that used in the CARRÉ study.

Results: After 20 years of follow up 118 patients (33\%) developed at least one CV event in the Carré group. Mean (SD) follow up time was 11 (6) years with a total of 3,500 years at risk and an incidence rate of 3.4 per 100 patient-years; this is slightly up from the figure reported at 15 years, i.e. 3.2 per 100 patient-years. A CV event-free survival curve is shown in figure 1. After 30 years of follow up, 295 participants of the Hoorn study had developed a CV event, during a mean follow up time 20 (8) years. Total time at risk was 50,000 years, with an incidence rate of $0.6 \mathrm{CV}$ events per 100 patient years.

Conclusion: In our cohort the incidence rate of $\mathrm{CV}$ events in RA patients has remained consistently high when compared with the general population, despite better control of RA inflammation in recent years. This again confirms the need for timely CVD-risk screening and management.

REFERENCES:

[1] Agca R, Hopman L, Laan KJC, van Halm VP, Peters MJL, Smulders YM, et al. Cardiovascular Event Risk in Rheumatoid Arthritis Compared with Type 2 Diabetes: A 15-year Longitudinal Study. J Rheumatol. 2020;47(3):316-24.

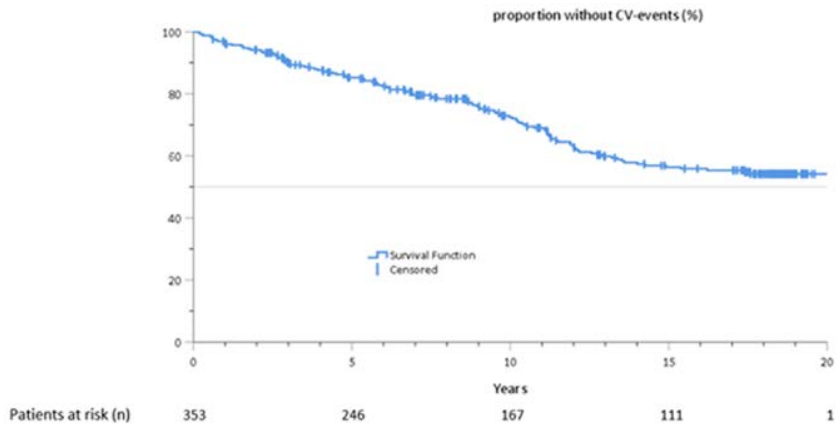

Figure 1. Survival curve of participants with rheumatoid arthritis. $\mathrm{RA}=$ rheumatoid arthritis
Disclosure of Interests: None declared

DOI: 10.1136/annrheumdis-2021-eular.1744

\section{POS0214 \\ ASSOCIATION BETWEEN C-REACTIVE PROTEIN AND 10-YEAR RISK OF CARDIOVASCULAR DISEASE IN RHEUMATOID ARTHRITIS USING THE ERS-RA SCORE: A CROSS-SECTIONAL ANALYSIS OF THE CORDIS COHORT}

G. L. Erre ${ }^{1}$, F. Cacciapaglia ${ }^{2}$, G. Sakellariou ${ }^{3}$, A. Manfredi ${ }^{4}$, E. Bartoloni Bocci $^{5}$, O. Viapiana ${ }^{6}$, M. Fornaro ${ }^{2}$, M. Dessi ${ }^{7}$, A. A. Mangoni ${ }^{8}$, B. L. Palermo ${ }^{9}$, E. Gremese ${ }^{10}$, G. Cafaro ${ }^{5}$, V. Nucera ${ }^{11}$, C. Vacchi ${ }^{12}$, F. R. Spinelli ${ }^{13}$, F. Atzeni ${ }^{11}$ M. Piga ${ }^{14}$ on behalf of "Cardiovascular Obesity and Rheumatic Disease Study (CORDIS)" Group of the Italian Society of Rheumatology (SIR).. 'University and AOU of Sassari, Department of Medical, Surgical and Experimental Sciences, Sassari, Italy; ${ }^{2}$ University and AOU, Policlinico of Bari, Department of Emergency and Organs Transplantation, Bari, Italy; ${ }^{3}$ University of Pavia, Istituti Clinici Scientifici Maugeri IRCCS Pavia, Pavia, Italy; ${ }^{4}$ Azienda Ospedaliera Universitaria Policlinico di Modena, Unit of Rheumatology, Modena, Italy; ${ }^{5}$ University of Perugia, Department of Medicine and Surgery, Perugia, Italy; ${ }^{6}$ University of Verona, Dipartimento di Medicina, Verona, Italy; ${ }^{7}$ University of Cagliari, Department of Medical Sciences and Public Health, Cagliari, Italy; ${ }^{8}$ Flinders University, College of Medicine and Public Health, Adelaide, Australia; ${ }^{9}$ University of Pavia, IRCSS San Matteo, Pavia, Italy; ${ }^{10}$ Policlinico Gemelli, Università Cattolica del Sacro Cuore, Dipartimento di Scienze Mediche e Chirurgiche, Roma, Italy; ${ }^{11}$ University of Messina, Dipartimento di Medicina Clinica e Sperimentale, Messina, Italy; ${ }^{12}$ University of Modena and Reggio Emilia, Clinical and Experimental Medicine PhD Program, Modena, Italy; ${ }^{13}$ Università La Sapienza, Dipartimento di Scienze Cliniche Internistiche, Anestesiologiche e Cardiovascolari, Roma, Italy; ${ }^{14}$ University of Cagliari, Department of Medical Sciences and Public Health, Monserrato, Italy

Background: Rheumatoid arthritis (RA) is associated with an increased risk of atherosclerotic cardiovascular disease (CVD). The Expanded Cardiovascular Risk Prediction Score for Rheumatoid Arthritis (ERS-RA) estimates the 10-year risk of myocardial infarction, stroke or CVD-related death based on conventional and RA-specific (clinical disease activity index, CDAl, disease duration, glucocorticoid use) risk factors (1).

Objectives: We evaluated the associations between ERS-RA 10-year risk of CVD, high-sensitivity C-reactive protein (hs-CRP) concentrations, and pharmacological treatment in 1,251 RA patients collected by the "Cardiovascular Obesity and Rheumatic Disease Study (CORDIS)" group of the Italian Society of Rheumatology (SIR)

Methods: We assessed independent associations between ERS-RA risk score and each relevant variable using multivariate regression (ENTER approach; listwise deletion analysis). Given the relatively high number of missing hs-CRP data $(n=385)$, regression analysis was also performed using multiple imputation (10 sets, Stata 16.1). Regression models were not adjusted for independent variables included in the ERS-RA score.

Results: Among 1,251 RA patients [mean (SD) age 60.4(9.3), range (40 80 ) years; $78 \%$ female; mean (SD) disease duration, 11.6(8) years; mean (SD) CDAI, 9(9); mean (SD) HAQ, 0.77(0.7); mean (SD) hs-CRP, 6.8(12) $\mathrm{mg} / \mathrm{L}$ ] the estimated 10-year CVD risk was $11.6(0.9) \%$ [mean (SD)]. Regarding treatment, 539(43\%) received glucocorticoids, 676(54\%) a biological or targeted synthetic disease-modifying anti-rheumatic drug (b/tsDMARD) (n missing=1), and $885(81 \%$ ) at least one conventional synthetic DMARD (csDMARD). Ninety-three (7.4\%) patients did not receive any treatment. After adjusting for the use of b/tsDMARD and csDMARD, hs-CRP concentrations were significantly associated with 10-year risk of CVD both in standard multiple regression ( $n=865$; coefficient $=0.005$ for each $10 \mathrm{mg} / \mathrm{L}$ hs-CRP increment, $95 \%$ confidence interval (0.000-0.100), $p=0.043$ ) and after multiple imputation $(n=1,251$; coefficient $=0.005$ for each $10 \mathrm{mg} / \mathrm{L}$ hs-CRP increment, $95 \%$ confidence interval $(0.000-0.114), p=0.035)$ (Table 1). This corresponds to an increase of 10 -year CV risk of $1 \%$ for every $20 \mathrm{mg} / \mathrm{L}$ increase in hs-CRP concentrations.

Conclusion: In a large cohort of RA patients, we observed a significant, positive, and independent association between hs-CRP concentrations and 10-year CV risk estimated by ERS-RA. The cross-sectional design of the study did not allow to establish a cause-effect relationship between hs-CRP and CV risk. Given that conventional $\mathrm{CV}$ risk factors and inflammation-related variables are accounted for in the ERS-RA risk score, other, unexplored, mechanisms may underlie the observed association between hs-CRP and CV risk.

\section{REFERENCES:}

[1] Solomon, D. H., et al. "Derivation and internal validation of an expanded cardiovascular risk prediction score for rheumatoid arthritis: a Consortium of Rheumatology Researchers of North America Registry Study." Arthritis \& rheumatology 67.8 (2015): 1995-2003. 